\title{
Delay-dependent stabilization of Lipschitz nonlinear systems
}

\author{
ARUNIMA MUKHERJEE*(D) and APARAJITA SENGUPTA \\ Department of Electrical Engineering, Indian Institute of Engineering Sciences and Technology, Shibpur, \\ Howrah 711103, India \\ e-mail: amukherjee966@gmail.com
}

MS received 5 February 2017; revised 5 September 2017; accepted 13 January 2018; published online 24 July 2018

\begin{abstract}
This work proposes an improvement over existing delay-dependent stabilization technique for linear systems having time-varying delays and norm-bounded uncertainties. The method is based on linear matrix inequalities (LMIs). Parameter tuning or iterative techniques are not required to get the required controller, if the proposed LMIs are satisfied. The method is also extended for systems having Lipschitz nonlinearities.
\end{abstract}

Keywords. Delay-rate-dependent stabilization; linear matrix inequality; lipschitz nonlinear systems; normbound parametric uncertainties.

\section{Introduction}

Time delays are naturally present in mostly all physical systems [1-3], namely chemical processes, communication systems, nuclear reactors, biological systems, aerospace applications, robotics, photovoltaic cells, etc.

Enormous work already exists on detrimental effects of time delay on stability [2] of linear systems. References [3-5] contain results on delay-independent stabilization, which is considered to be simple yet conservative, while [6-13] deal with synthesis problems on the less conservative delay-dependent stabilization techniques. On the other hand [14-23] discuss various stability criteria on permissible limits of time delay beyond which the system goes into instability. Before the work in $[8,9]$ the algorithms required the pair $(\mathrm{A}, \mathrm{B})$ to be stabilizable. Again [10] uses matrix inequalities for stabilization of time delay systems but since they are not linear, an iterative procedure is to be adopted to obtain the controller parameters. Also [12] proposes an approach based on widely used linear matrix inequalities (LMIs), but the drawback is that four parameters need to be tuned properly for desirable results. The technique in [13] is a design based entirely on LMI. Most of the work reported concentrates on the analysis of linear time delay systems, i.e., developing stability criteria for them, while less work is done on controller synthesis for time delay systems, i.e., stabilization. Hence, there is a need for further investigation to obtain techniques that yield controllers for stabilization without going into complex optimization routines and parameter tuning.

Also in the vast literature listed earlier, less attention is paid on stabilization of systems that contain nonlinearity along

*For correspondence with time delays. Practical time delay systems like biological ones, chemical processes and economic processes [3] are often nonlinear as well as uncertain. Soft computing techniques are used in references [24-28] and other techniques in [29-31] for nonlinear time delay systems. They yields controllers that are either dynamic or nonlinear functions of states, thus posing a difficulty in implementation.

Therefore the present work also addresses synthesis problems for Lipschitz nonlinear time delay systems. In the last decade a vast literature ([32-35] and references therein) is reported on observer design for such systems as this type of nonlinearity can represent a class of practical systems like robotic ones (trigonometric nonlinearity) or systems with square or cubic functions of states. Besides this, most nonlinear systems can be modelled as locally Lipschitz ones. However, fewer attempts are made to design controllers for uncertain time delay systems containing Lipschitz nonlinearity. This provides us the second motivation.

In this work, first an improved stabilizing controller for linear systems with time delay and norm-bound uncertainty is proposed. If feasible, the controller can be found by solving a few LMI conditions and does not require additional optimization or further parameter tuning. Table 1 contains a comparison of the results in existing and the present work. The control law obtained here is in the form of Linear State Variable Feedback (LSVF), which is simple to implement either through states or through observers. The work is then extended for time delay systems with Lipschitz nonlinearities. It is also shown with the help of an example that the nonlinearity in a system can be better handled by directly including its effect in the LMI, rather than assuming it to be a perturbation, which is a more common practice in literature $[36,37]$. 
Table 1. Permissible maximum delay limit $\tau_{\max }$.

\begin{tabular}{lc}
\hline References & Maximum delay limit $\tau_{\max }$ \\
\hline$[10]$ & 0.45 \\
{$[11]$} & 0.58 \\
{$[13]$} & 0.68 \\
Proposed Theorem 2 & 0.78 \\
\hline
\end{tabular}

The paper is organized as follows. Section 1 contains the mathematical preliminaries required for the derivations. Section 2 gives the problem statement. Section 3 provides the main results, where theorems yielding a stabilizing controller for linear systems as well as Lipschitz nonlinear systems with time delay are given, with their respective proofs. The theorems are then further extended for systems containing norm-bounded uncertainty. The paper also contains numerical examples to show the applicability of the proposed theory, which are given in section 4 .

In this paper, $\|\cdot\|$ represents Euclidean norm. $\mathbb{R}^{+}$represents the set of all positive real numbers. $\mathbb{R}^{n \times n}$ represents the matrix space of real numbers of $n \times n$ dimensions. $\mathbb{S}^{n+}$ represents the matrix space of all positive-definite matrices of $n \times n$ dimensions and $\mathbb{S}^{n}$ represents the matrix space of all symmetric matrices of $n \times n$ dimensions.

\section{Preliminaries}

Lemma 1 [38] For given matrices $M$ and $N$ with appropriate dimensions and a norm-bound time-varying matrix $F(t)$, such that $\|F(t)\| \leq 1$, we have the following condition:

$$
M F(t) N+M^{T} F(t)^{T} N^{T} \leq \varepsilon M M^{T}+\varepsilon^{-1} N^{T} N
$$

where $\varepsilon$ is a positive scalar.

Lemma 2 Given matrices $\phi=\phi^{T}, D, E$ and $R=R^{T} \succ 0$ of appropriate dimensions

$$
\phi+H F(t) E+E^{T} F(t)^{T} H^{T} \prec 0
$$

for all $F(t)$ satisfying $F(t)^{T} F(t) \prec R$, if and only if there exists some positive scalar $\lambda$ such that

$$
\phi+\lambda H H^{T}+\lambda^{-1} E^{T} R E \prec 0 .
$$

\section{Problem formulation}

Consider the linear system description

$$
\begin{aligned}
& \dot{x}(t)=A x(t)+A_{1} x(t-\delta(t))+B u(t) \\
& x(t)=\Upsilon(t), t \in[-\tau, 0]
\end{aligned}
$$

with $A, A_{1} \in \mathbb{R}^{n \times n}$ and $B \in \mathbb{R}^{n \times 1}$, the system matrices containing $\delta(t)$, a time-varying delay, satisfying

$$
0 \leq \delta(t) \leq \tau
$$

and

$$
\dot{\delta}(t) \leq \mu \leq 1
$$

$\Upsilon(t)$ being an initial function. Here the objective is to stabilize the linear time delay system (3) using an LSVF control $u(t)$, where

$$
u(t)=K x(t)
$$

and $K$ is the LSVF controller gain matrix. Results are subsequently extended to time delay systems with Lipschitz nonlinearity.

\section{Main results}

\subsection{Stabilization of a linear time delay system}

Theorem 1 The system described by (3) with time-varying delay $\delta(t)$ satisfying (4) and (5) can be stabilized using a memoryless controller given by $K=G H^{-1}$ if $H, Q$, $\in \mathbb{S}^{n+}, X \in \mathbb{S}^{2 n+}$ and $Y, T, N_{1}, N_{2} \in \mathbb{S}^{n}$ exist such that the following two LMIs are satisfied:

LMI-1

$$
\phi_{L T D 1}=\left[\begin{array}{ccc}
(1,1) & (1,2) & \tau\left(H A^{T}+G^{T} B^{T}\right) \\
\star & (2,2) & \tau H A_{1}^{T} \\
\star & \star & -\tau I
\end{array}\right] \prec 0
$$

$L M I-2$

$$
\left[\begin{array}{ccc}
x_{11} & x_{12} & N_{1} \\
\star & x_{22} & N_{2} \\
\star & \star & I
\end{array}\right] \succ 0
$$

Here $(1,1)=H A^{T}+A H+G^{T} B^{T}+B G+Q+\tau x_{11}+2 Y$, $(1,2)=A_{1} H-Y+T+\tau x_{12},(2,2)=-(1-\mu) Q+\tau x_{22}$ $-2 T$ and $X$ is partitioned as $\left[\begin{array}{cc}x_{11} & x_{12} \\ \star & x_{22}\end{array}\right]$.

Proof Let the Lyapunov-Krasovskii functional candidate be given by $v(x(t))$ :

$$
v(x(t))=v_{1}+v_{2}+v_{3} .
$$

Here

$$
\begin{aligned}
& v_{1}=x^{T}(t) P x(t), \\
& v_{2}=\int_{t-\delta(t)}^{t} x^{T}(s) R x(s) d s, \\
& v_{3}=\int_{-\tau}^{0} \int_{t+\theta)}^{t} \dot{x}^{T}(s) \dot{x}(s) d s d \theta .
\end{aligned}
$$

Using (3)-(5) the following values of derivatives of $v_{i}$ are found for $i=1,2,3$, and using standard manipulations 


$$
\dot{v}_{1}=\zeta^{T}\left[\begin{array}{cc}
(A+B K)^{T} P+P(A+B K) & P A_{1} \\
A_{1} P & 0
\end{array}\right] \zeta
$$

where $\zeta=\left[\begin{array}{ll}x^{T}(t) & x^{T}(t-\delta(t))\end{array}\right]^{T}$,

$$
\begin{gathered}
\dot{v}_{2}=x^{T}(t) R x(t)-(1-\dot{\delta}(t)) x^{T}(t-\delta(t)) R x(t-\delta(t)), \\
\dot{v}_{3}=\dot{x}^{T}(t) \dot{x}(t) \tau-\int_{t-\tau}^{t} \dot{x}^{T}(s) \dot{x}(s) d s
\end{gathered}
$$

Also consider the Leibniz-Newton formula

$$
\left[x(t)-x(t-\delta(t))-\int_{t-\delta(t)}^{t} \dot{x}(s) d s\right]=0 .
$$

Based on this formula and using symmetric matrices $\mathrm{U}$ and $\mathrm{V}$, we get the relation [19]

$$
2\left[x^{T}(t) U+x^{T}(t-\delta(t)) V\right] \times \psi=0
$$

where $\psi=\left[x(t)-x(t-\delta(t))-\int_{t-\delta(t)}^{t} \dot{x}(s) d s.\right]$

Using any positive semi-definite matrix $S=\left[\begin{array}{cc}s_{11} & s_{12} \\ \star & s_{22}\end{array}\right]$, with the condition given by (4) the following inequality is obtained:

$$
\tau \zeta^{T} S \zeta-\int_{t-\delta(t)}^{t} \zeta^{T} S \zeta d s \succeq 0 .
$$

Combining (10)-(12), (14) and (15) we get

$$
\dot{v}\left(x_{t}\right) \preceq \zeta^{T}\left[\begin{array}{cc}
(1,1) & (1,2) \\
\star & (2,2)
\end{array}\right] \zeta-\int_{t-\delta(t)}^{t} \bar{\zeta}^{T}\left[\begin{array}{cc}
S & \rho \\
\rho^{T} & I
\end{array}\right] \bar{\zeta} d s
$$

where $\quad \bar{\zeta}=\left[\begin{array}{lll}x^{T}(t) & x^{T}(t-\delta(t)) & \dot{x}^{T}(s)\end{array}\right]^{T}, \quad \rho=\left[\begin{array}{c}U \\ V\end{array}\right]$, $(1,1)=(A+B K)^{T} P+P(A+B K)+\tau(A+B K)^{T}(A+$ $B K)+R+\tau s_{11}+2 U,(1,2)=P A_{1}+\tau(A+B K)^{T}$ $A_{1}-U+V+\tau s_{12}, \quad(2,2)=-(1-\mu) R+\tau A_{1}^{T} A_{1}-2 V+$ $\tau s_{22}$. Relation (16) necessitates the following two conditions for $\dot{v} \prec 0$ :

I

$$
\left[\begin{array}{cc}
(1,1) & (1,2) \\
\star & (2,2)
\end{array}\right] \prec 0
$$

and

II

$$
\left[\begin{array}{cc}
S & \rho \\
\rho^{T} & I
\end{array}\right] \succ 0
$$

with $(1,1),(1,2),(2,2)$ and $\rho$ as in (16). Now consider the inequality

$$
\left[\begin{array}{ccc}
\Xi_{11} & \Xi_{12} & \tau\left(A^{T}+K^{T} B^{T}\right) \\
\star & \Xi_{22} & \tau A_{1}^{T} \\
\star & \star & -\tau I
\end{array}\right] \prec 0 .
$$

Here $\quad \Xi_{11}=(A+B K)^{T} P+P(A+B K)+R+\tau s_{11}+2 U$, $\Xi_{12}=P A_{1}-U+V+\tau s_{12}, \quad \Xi_{22}=-(1-\mu) R+\tau s_{22}-2 V$.

Using the Schur Complement Lemma it can be easily shown that (19) is equivalent to (17). However, (19) is not an LMI; hence, to convert it into an LMI, (19) is pre- and post-multiplied with a positive-definite matrix given by $\operatorname{diag}\left\{P^{-1}, P^{-1}, P^{-1}\right\}$. Defining new variables as $P^{-1}:=H$, $K P^{-1}:=G, P^{-1} R P^{-1}:=Q, P^{-1} s_{11} P^{-1}:=x_{11}, P^{-1} s_{12} P^{-1}$ : $=x_{12}, P^{-1} s_{22} P^{-1}:=x_{22}, P^{-1} U P^{-1}:=Y, P^{-1} V P^{-1}:=T$, the inequality (7) given in Theorem 1 is obtained.

Next inequality (18) is considered, which on preand post-multiplication with $\operatorname{diag}\left\{P^{-1}, P^{-1}, P^{-1}\right\}$ gives (20):

$$
\left[\begin{array}{ccc}
P^{-1} s_{11} P^{-1} & P^{-1} s_{12} P^{-1} & P^{-1} U P^{-1} \\
\star & P^{-1} s_{22} P^{-1} & P^{-1} V P^{-1} \\
\star & \star & P^{-1} P^{-1}
\end{array}\right] \succ 0
$$

Note: The condition given by (20) is not an LMI due to the presence of square terms in $P^{-1}$. Using Schur's complement (20) yields the following conditions:

$$
P^{-1} P^{-1} \succ 0
$$

and

$$
\Lambda-\left[\begin{array}{l}
P^{-1} U \\
P^{-1} V
\end{array}\right]\left[\begin{array}{ll}
U P^{-1} & V P^{-1}
\end{array}\right] \succ 0
$$

where $\Lambda=\left[\begin{array}{cc}P^{-1} s_{11} P^{-1} & P^{-1} s_{12} P^{-1} \\ \star & P^{-1} s_{22} P^{-1}\end{array}\right]$.

Inequality (22) is true only when there exists some $\varphi>1$ such that (23) is true:

$$
\Lambda \succ \varphi\left[\begin{array}{l}
P^{-1} U \\
P^{-1} V
\end{array}\right]\left[\begin{array}{ll}
U P^{-1} & V P^{-1}
\end{array}\right] .
$$

Equivalently

$$
\Lambda-\left[\begin{array}{l}
P^{-1} U c \\
P^{-1} V c
\end{array}\right]\left[\begin{array}{ll}
c U P^{-1} & c V P^{-1}
\end{array}\right] \succ 0 .
$$

Here, $c=\sqrt{\varphi}$.

Defining new variables $P^{-1} U c:=N_{1}$, and $P^{-1} V c:=N_{2}$, the inequality (8) given in Theorem 1 is obtained, which completes the proof. 


\subsection{Stabilization of a linear time delay system with norm-bound uncertainty}

No models can represent real physical systems exactly. In order to incorporate more practical information, time delay systems with structured uncertainty (25) are considered in this section. The system has norm-bound uncertainty.

$$
\begin{aligned}
\dot{x}(t) & =\bar{A} x(t)+\bar{A}_{1} x(t-\delta(t))+B u(t) \\
x(t) & =\Upsilon(t), t \in[-\tau, 0]
\end{aligned}
$$

where $\bar{A}$ and $\bar{A}_{1}$ contain norm-bounded uncertainty $F(t)$ and have structures given by

$$
\bar{A}=A+D F(t) E a
$$

and

$$
\bar{A}_{1}=A_{1}+D F(t) E a_{1} .
$$

The objective is to stabilize (25) by an LSVF control law of the form (6). Theorem 2 yields such a controller.

Theorem 2 For the system described in (25), if $H$, $Q \in \mathbb{S}^{n+}, X \in \mathbb{S}^{2 n+}$ and $Y, T, N_{1}, N_{2} \in \mathbb{S}^{n}$ exist such that the following two LMIs are satisfied, then a memoryless controller given by $K=G H^{-1}$ stabilizes the system:

LMI-1

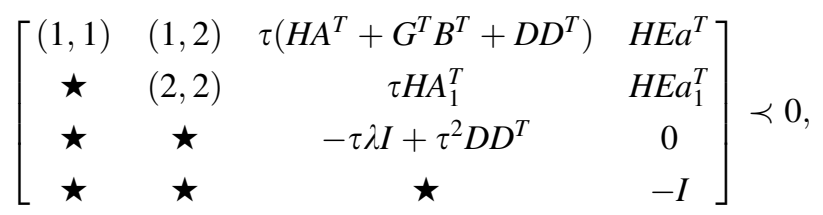

$L M I-2$

$$
\left[\begin{array}{ccc}
x_{11} & x_{12} & N_{1} \\
\star & x_{22} & N_{2} \\
\star & \star & I
\end{array}\right] \succ 0 .
$$

Here $(1,1)=H A^{T}+A H+G^{T} B^{T}+B G+Q+\tau x_{11}+2 Y+$ $D D^{T},(1,2)=A_{1} H-Y+T+\tau x_{12},(2,2)=-(1-\mu) Q+$ $\tau x_{22}-2 T$ and $X$ is partitioned as $\left[\begin{array}{cc}x_{11} & x_{12} \\ \star & x_{22}\end{array}\right]$.

Proof Consider Theorem 1, in (7). $A$ and $A_{1}$ are replaced with their uncertain counterparts $\bar{A}$ and $\bar{A}_{1}$, as in (26) and (27). Now the new inequality, after altering (7) to include uncertainty, is given by the relation

$$
\phi_{L T D 1}+\Sigma_{1} \prec 0 .
$$

Here

$$
\begin{aligned}
\Sigma_{1} & =\left[\begin{array}{ccc}
H \delta A^{T}+\delta A H & \delta A_{1} H & \tau H \delta A^{T} \\
H \delta A_{1}^{T} & 0 & \tau H \delta A_{1}^{T} \\
\tau \delta A H & \tau \delta A_{1} H & 0
\end{array}\right] \\
\delta A & =D F(t) E a \\
\delta A_{1} & =D F(t) E a_{1}
\end{aligned}
$$

using Lemma 2 and carrying out some standard manipulations on (30), the matrix inequality given in (28) in Theorem 2 is obtained. The second inequality (29) can be obtained in the same way as in Theorem 1.

Remark 1 If in (3) and (25) the system description is completely known, then the controller $K$ for the individual systems can be obtained directly using Theorems 1 and 2, respectively. If the respective LMI constraints are satisfied, then such $K$ s can directly stabilize (3) and (25). No additional parameter tuning or further optimization is required and hence the proposed theorems are easier compared with those in $[10,12]$.

Remark 2 For a system (as in (3)), the maximum allowable delay limit $\tau_{\max }$ can be found by the following method.

\section{Iterative method for obtaining $\tau_{\max }$}

Here (7) is not linear in $\tau$; thus, direct maximization w.r.t this variable is not possible. Hence, an iterative method is to be used; $\tau_{\max }$ is found iteratively by increasing the value of $\tau$ starting from a low value and checking the feasibility of LMI constraints (7) and (8), till the maximum $\tau_{\max }$ is reached. Beyond this $\tau=\tau_{\max }$, the solution to LMIs (7) and (8) becomes infeasible.

Remark 3 Remark 2 can be easily extended for (25) to find its $\tau_{\max }$. The only difference is the use of LMI constraints given in (28) and (29) instead of (7) and (8) in Remark 2.

\subsection{Stabilization of Lipschitz nonlinear systems with time delay}

In this section, the work in section 3.1 is extended to systems having Lipschitz nonlinearity.

System description:

$$
\begin{aligned}
& \dot{x}(t)=A x(t)+A_{1} x(t-\delta(t))+B u(t)+g(x), \\
& x(t)=\Upsilon(t), t \in[-\tau, 0] .
\end{aligned}
$$

Here $\delta(t)$ is a time-varying delay satisfying (4) and (5). Function $g(x)$ is a Lipschitz nonlinear function of state vector $x$, and satisfies the inequality given by

$$
g^{T}(x) g(x) \preceq \gamma^{2} x^{T}(t) x(t)
$$

and also $g(0)=0$. Here $\gamma$ is the Lipschitz constant. Attempt is made to derive a control law (6) for the system in (31). Theorem 1 is extended to Theorem 3 to accommodate the 
nonlinearity as in (32). This nonlinearity commonly occurs in physical systems in cubic, square or trigonometric forms.

Theorem 3 The system described by (31) with timevarying delay $\delta(t)$ can be stabilized using a memoryless controller given by $K=G H^{-1}$ if there exist $H, Q \in \mathbb{S}^{n+}$, $X \in \mathbb{S}^{2 n+}$ and $Y, T, N_{1}, N_{2} \in \mathbb{S}^{n}$, such that the following two LMIs are satisfied:

LMI-1

$$
\phi_{1}=\left[\begin{array}{ccccc}
(1,1) & (1,2) & I & \tau\left(H A^{T}+G^{T} B^{T}\right) & \gamma H \\
\star & (2,2) & 0 & \tau H A_{1}^{T} & 0 \\
\star & \star & -I & \tau I & 0 \\
\star & \star & \star & -\tau I & 0 \\
\star & \star & \star & \star & -I
\end{array}\right] \prec 0
$$

$L M I-2$

$$
\left[\begin{array}{ccc}
s_{11} & s_{12} & N_{1} \\
\star & s_{22} & N_{2} \\
\star & \star & I
\end{array}\right] \succ 0
$$

where $(1,1)=H A^{T}+A H+G^{T} B^{T}+B G+Q+\tau x_{11}+2 Y$, $(1,2)=A_{1} H-Y+T+\tau x_{12}, \quad(2,2)=-(1-\mu) Q+$ $\tau x_{22}-2 T$ and $X$ is partitioned as $\left[\begin{array}{cc}x_{11} & x_{12} \\ \star & x_{22}\end{array}\right]$.

Proof Consider the same Lyapunov-Krasovskii functional as given in (9). For this, $\dot{v}_{2}$ and $\dot{v}_{3}$ are the same as those given in (11) and (12), respectively, while $\dot{v}_{1}$ is given as follows:

$$
\dot{v}_{1}=\epsilon^{T}\left[\begin{array}{ccc}
(A+B K)^{T} P+P(A+B K) & P A_{1} & P \\
A_{1} P & 0 & 0 \\
P & 0 & 0
\end{array}\right] \epsilon
$$

where $\epsilon^{T}=\left[\begin{array}{lll}x^{T}(t) & x^{T}(t-\delta(t)) & g^{T}(x)\end{array}\right]$. Also, from (32)

$$
0 \preceq-g^{T}(x) g(x)+\gamma^{2} x^{T}(t) x(t) .
$$

Combining (35), (11), (12), (36), (14) and (15) we get

$$
\dot{v}\left(x_{t}\right) \preceq \epsilon^{T}\left[\begin{array}{ccc}
(1,1) & (1,2) & P+(A+B K)^{T} \tau \\
\star & (2,2) & A_{1}^{T} \tau \\
\star & \star & \tau I-I
\end{array}\right] \epsilon-\varrho
$$

$$
\begin{aligned}
& \text { where } \varrho=\int_{t-\delta(t)}^{t} \bar{\zeta}^{T}\left[\begin{array}{cc}
S & \rho \\
\rho^{T} & I
\end{array}\right] \bar{\zeta} d s, \\
& \begin{aligned}
(1,1) & =(A+B K)^{T} P+P(A+B K)+\tau(A+B K)^{T}(A+ \\
B K)+R & +\tau s_{11}+2 U+\gamma^{2} I, \\
(1,2) & =P A_{1}+(A+B K)^{T} A_{1} \tau-U+T+\tau s_{12}, \\
(2,2) & =-(1-\mu) R+A_{1}^{T} A_{1} \tau-2 T+\tau s_{22} .
\end{aligned}
\end{aligned}
$$

From (37), $\dot{v} \prec 0$ if

I

$$
\left[\begin{array}{ccc}
(1,1) & (1,2) & P+(A+B K)^{T} \tau \\
\star & (2,2) & A_{1}^{T} \tau \\
\star & \star & \tau I-I
\end{array}\right] \prec 0
$$

and

II

$$
\left[\begin{array}{ll}
S & \rho \\
\rho^{T} & I
\end{array}\right] \succ 0
$$

Consider the inequality

$$
\left[\begin{array}{ccccc}
\Xi_{11} & \Xi_{12} & \Xi_{13} & \gamma I & \tau\left(A^{T}+K^{T} B^{T}\right) \\
\star & \Xi_{22} & 0 & 0 & \tau A_{1}^{T} \\
\star & \star & -I & 0 & \tau I \\
\star & \star & \star & -I & 0 \\
\star & \star & \star & \star & -\tau I
\end{array}\right] \prec 0
$$

Here $\Xi_{1,1}=(A+B K)^{T} P+P(A+B K)+R+\tau s_{11}+2 U$, $\Xi_{1,2}=P A_{1}-U+V+\tau s_{12}, \quad \Xi_{1,3}=P, \quad \Xi_{2,2}=-(1-$ $\mu) R+\tau s_{22}-2 T$. Using the Schur complement Lemma, it can be easily shown that (40) is equivalent to (38). However, as before (40) is not an LMI; hence, to obtain LMI condition we pre- and post-multiply the inequality with a positive-definite matrix given by $\operatorname{diag}\left\{P^{-1}, P^{-1}, I, I, I\right\}$. Defining new variables as $P^{-1}:=H, \quad K P^{-1}:=G$, $P^{-1} R P^{-1}:=Q, \quad P^{-1} s_{11} P^{-1}:=x_{11}, \quad P^{-1} s_{12} P^{-1}:=x_{12}$, $P^{-1} s_{22} P^{-1}:=x_{22}, P^{-1} U P^{-1}:=Y \quad \& \quad P^{-1} V P^{-1}:=T$, the inequality (33) given in Theorem 3 is obtained. ${ }^{1}$

\subsection{Stabilization of Lipschitz nonlinear systems with time delay and norm-bound uncertainty}

Finally consider the system given by (41) with Lipschitz nonlinearity, norm-bound uncertainty as well as time delay:

$$
\begin{aligned}
& \dot{x}(t)=\bar{A} x(t)+\bar{A}_{1} x(t-\delta(t))+B u(t)+g(x) \\
& x(t)=\Upsilon(t), t \in[-\tau, 0]
\end{aligned}
$$

with $\bar{A}$ and $\bar{A}_{1}$ as in (26) and (27), respectively. The controller in (6) may be obtained for (41) from Theorem 4.

Theorem 4 For the system described in (41), if there exist $H, Q \in \mathbb{S}^{n+}, X \in \mathbb{S}^{2 n+}, Y, T, N_{1}, N_{2} \in \mathbb{S}^{n}$ and $\lambda \in \mathbb{R}^{+}$such that the following two LMIs are satisfied, then a memoryless controller given by $K=G H^{-1}$ can stabilize the system.

\footnotetext{
${ }^{1}$ The second inequality (34) can be obtained in the same way as in Theorem 1.
} 
LMI-1

$$
\left[\begin{array}{cccccc}
(1,1) & (1,2) & I \lambda & (1,4) & \gamma H & H E a^{T} \\
\star & (2,2) & 0 & \tau H A_{1}^{T} & 0 & H E a_{1}^{T} \\
\star & \star & -I \lambda & \tau \lambda I & 0 & 0 \\
\star & \star & \star & -\tau \lambda I+\tau^{2} D D^{T} & 0 & 0 \\
\star & \star & \star & \star & -I \lambda & 0 \\
\star & \star & \star & \star & \star & -I
\end{array}\right] \prec 0
$$

LMI-2

$$
\left[\begin{array}{ccc}
x_{11} & x_{12} & N_{1} \\
\star & x_{22} & N_{2} \\
\star & \star & I
\end{array}\right] \succ 0
$$

where $(1,1)=H A^{T}+G^{T} B^{T}+A H+B G+Q+\tau x_{11}+Y+$ $2 Y+D D^{T}, \quad(1,2)=A_{1} H-Y+T+\tau x_{12}, \quad(1,4)=$ $\tau\left(H A^{T}+G^{T} B^{T}+D D^{T}\right), \quad(2,2)=-(1-\mu) Q+\tau x_{22}-$ $(T+T)$ and $X$ is partitioned as: $\left[\begin{array}{cc}x_{11} & x_{12} \\ \star & x_{22}\end{array}\right]$.

Proof Consider $\phi_{1}$ as in Theorem 3 given in (33). $A$ and $A_{1}$ are replaced with their uncertain counterparts $\bar{A}$ and $\bar{A}_{1}$. They are then expanded according to (26) and (27). Now the new inequality, after altering (33) to include uncertainty, is given as follows:

$$
\phi_{1}+\left[\begin{array}{ccccc}
H \delta A^{T}+\delta A H & \delta A_{1} H & 0 & \tau H \delta A^{T} & 0 \\
H \delta A_{1}^{T} & 0 & 0 & \tau H \delta A_{1}^{T} & 0 \\
0 & 0 & 0 & 0 & 0 \\
\tau \delta A H & \tau \delta A_{1} H & 0 & 0 & 0 \\
0 & 0 & 0 & 0 & 0
\end{array}\right] \prec 0
$$

Using Lemma 2 and some manipulations on (44), (42) in Theorem 4 is obtained. The second inequality (43) can be obtained in the same way as in Theorem 3.

Remark 4 Remarks 1 and 2 can easily be extended for systems as in (31) and (41); hence, no separate rules for them are given.

Corollary 1 Consider the system

$$
\begin{aligned}
& \dot{x}(t)=\bar{A} x(t)+\bar{A}_{1} x(t-\delta(t))+g(x) \\
& x(t)=\Upsilon(t), t \in[-\tau, 0]
\end{aligned}
$$

with $\bar{A}$ and $\bar{A}_{1}$ as in (26) and (27), respectively. Note that this is system (41) without controller $K$.

For the system described in (45), if there exist $P$, $Q \in \mathbb{S}^{n+}, X \in \mathbb{S}^{2 n+}, Y, T \in \mathbb{S}^{n}$ and $\lambda \in \mathbb{R}^{+}$such that the following two LMIs are satisfied, then the system is asymptotically stable, for a given value of $\tau$.
LMI-1

$$
\left[\begin{array}{ccccc}
(1,1) & (1,2) & P & \tau \lambda A^{T} & P D \\
\star & (2,2) & 0 & \tau \lambda A_{1}^{T} & 0 \\
\star & \star & -I \lambda & \tau \lambda I & 0 \\
\star & \star & \star & -\tau \lambda I+\tau \lambda D & \\
\star & \star & \star & \star & -I
\end{array}\right] \prec 0
$$

LMI-2

$$
\left[\begin{array}{ccc}
x_{11} & x_{12} & Y \\
\star & x_{22} & T \\
\star & \star & I
\end{array}\right] \succ 0
$$

where

$$
(1,1)=A^{T} P+P A+Q+\tau x_{11}+2 Y+\lambda \gamma^{2} I+
$$
$E a^{T} E a, \quad(1,2)=P A_{1}-Y+T+\tau x_{12}+E a^{T} E a_{1}, \quad(2,2)=$ $-(1-\mu) Q+\tau x_{22}-(T+T)+E a_{1}^{T} E a_{1}$ and $X$ is partitioned as $\left[\begin{array}{cc}x_{11} & x_{12} \\ \star & x_{22}\end{array}\right]$.

Stability analysis of (45) for known values of $\tau$ can be performed using this corollary. The proof follows in same line as in Theorem 4; hence, it is omitted for brevity.

\section{Simulation results}

For checking the applicability of the proposed theorems two numerical examples are discussed here.

Example 1 Here Theorem 2 is applied to a numerical example taken from the literature. Consider (25) with

$$
\begin{aligned}
& A=\left[\begin{array}{ll}
0 & 0 \\
0 & 1
\end{array}\right], \quad A_{1}=\left[\begin{array}{cc}
-2 & -0.5 \\
0 & -1
\end{array}\right], \quad B=\left[\begin{array}{l}
0 \\
1
\end{array}\right] \\
& E a=\left[\begin{array}{ll}
1 & 0 \\
0 & 1
\end{array}\right], \quad E a 1=\left[\begin{array}{ll}
1 & 0 \\
0 & 1
\end{array}\right], \quad D=\left[\begin{array}{cc}
0.2 & 0 \\
0 & 0.2
\end{array}\right] .
\end{aligned}
$$

Note that for this example the maximum permissible delay limit $\tau_{\max }$ for a stable system is found using Theorem 2 and the iterative method described in Remarks 2,3 and is given in table 1 . The results of the proposed Theorem 2 is compared to existing ones.

Theorem 2 is based on delay-rate-dependent criterion as well as free matrices as compared with the existing ones, which do not use them simultaneously. Hence, delay limit is seen to improve with the proposed theorem. The controller $K$ for this example is given by the relation

$$
K=\left[\begin{array}{ll}
0.0731 & -4.3697
\end{array}\right] \text {. }
$$

Example 2 Consider the system inspired by [37] with time-varying delay $\delta(t)$ satisfying (4) and (5): 
Table 2. Permissible delay $\tau$.

\begin{tabular}{|c|c|c|c|c|c|c|}
\hline \multirow[b]{3}{*}{$\alpha$} & \multicolumn{3}{|c|}{$\mu=0.5, \beta=0.1$} & \multicolumn{3}{|c|}{$\mu=0.9, \beta=0.1$} \\
\hline & \multicolumn{3}{|c|}{$\tau$} & \multicolumn{3}{|c|}{$\tau$} \\
\hline & {$[37]$} & Corollary 1 & Theorem 4 & {$[37]$} & Corollary 1 & Theorem 4 \\
\hline 0 & 1.4 & 1 & 1.1 & 1.2 & 0.95 & 1.1 \\
\hline 0.1 & 1.2 & 0.93 & 1.1 & 1.2 & 0.86 & 1.06 \\
\hline 0.5 & N.F. & 0.89 & 1.08 & N.F. & 0.79 & 1 \\
\hline 0.75 & N.F. & 0.85 & 1.02 & N.F. & .7 & 1 \\
\hline 0.9 & N.F. & N.F. & 1 & N.F. & N.F. & 0.96 \\
\hline
\end{tabular}

N.F. $=$ not feasible

$$
\begin{aligned}
\dot{x}(t) & =A x(t)+A_{1} x_{\tau}+f(x(t), t)+g\left(x_{\tau}, t\right)+B u \\
x(t) & =\Upsilon(t), t \in[-\tau, 0] \\
x_{\tau} & =x(t-\delta(t)) .
\end{aligned}
$$

Here $f(x(t), t)$ and $g\left(x_{\tau}, t\right)$ are nonlinear perturbations obeying inequalities given by

$$
\begin{aligned}
f^{T}(x(t), t) f(x(t), t) & \preceq \alpha^{2} x^{T}(t) F^{T} F x(t) \\
g^{T}\left(x_{\tau}, t\right) g\left(x_{\tau}, t\right) & \preceq \beta^{2} x_{\tau}^{T} G^{T} G x_{\tau}
\end{aligned}
$$

where $A=\left[\begin{array}{cc}-1.2 & 0.1 \\ -0.1 & -1\end{array}\right], A_{1}=\left[\begin{array}{cc}-0.6 & 0.7 \\ -1 & -0.8\end{array}\right], B=\left[\begin{array}{l}0 \\ 1\end{array}\right]$ and $F=G=I$.

This system can be assumed to be a system with Lipschitz nonlinearity under the condition $F=G=I$. The results obtained from Corollary 1, Theorem 4 and [37] are compared in table 2

Remark 5 In table 2, from rows 3, 4 and 5 it is evident that more amount of nonlinearity may be accommodated through the newly proposed method. The maximum allowable value of $\alpha$ is 0.75 out of Corollary 1 and with Theorem 4 it is 0.9 as compared with 0.1 in [37].

Remark 6 The time response of the system also becomes better with a feedback and this is evident from figures 1 and 2; the plots, respectively, show the responses of the uncertain nonlinear time delay systems with and without feedback.

Attributes of delay-dependent and delay-rate-dependent controller design method

Consider Example 2. For different parameters ( $\tau, \alpha$ and $\mu$ ), peak of norm of the control effort, i.e., 'peak $\|K x\|$ ', is calculated and plotted in figures 3 and 4 .

In figure 3, a relationship between peak of $\|K x\|$ and $\alpha$ can be seen. Here it is clear that the control effort increases with increase in $\alpha$, with $\tau$ and $\mu$ being constants for each curve. Moreover, here, it can also be seen that with an

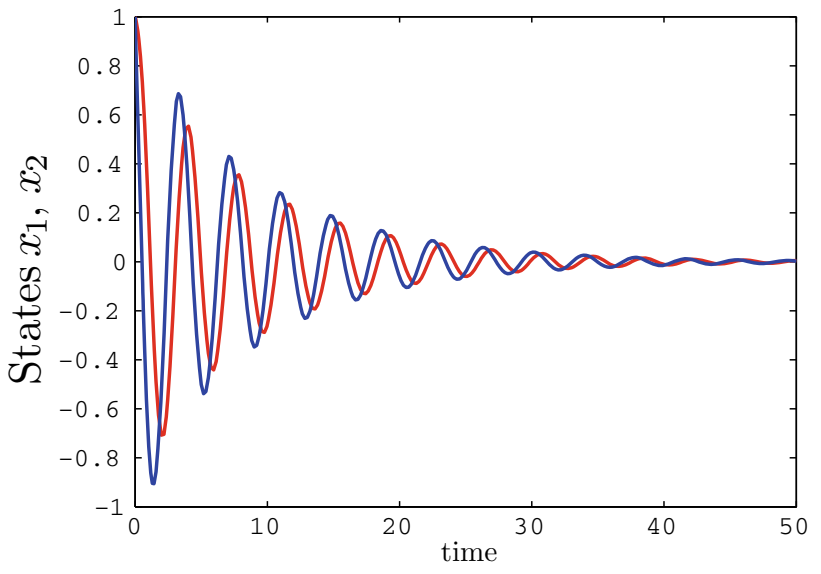

Figure 1. Response for the nonlinear time delay system without feedback.

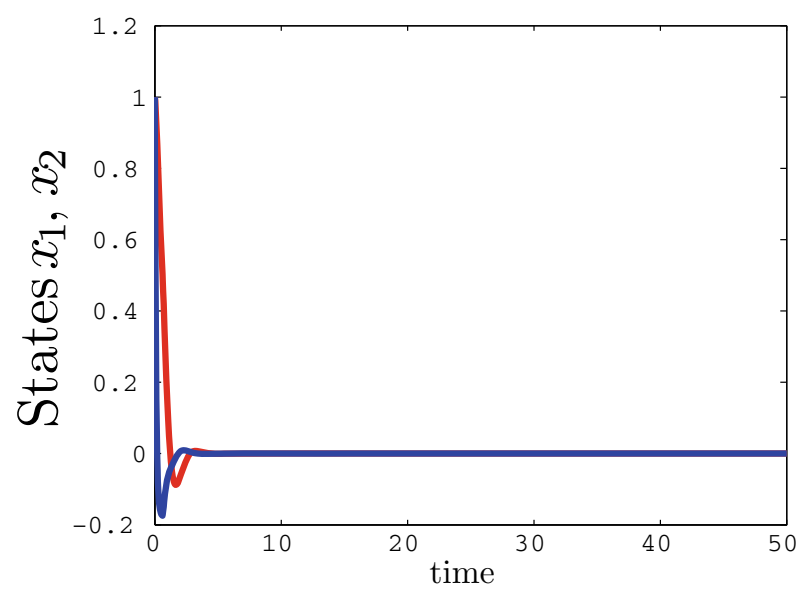

Figure 2. Response for the nonlinear time delay system with feedback.

increase in $\tau$, the control input increases for a particular $\alpha$. Thus the control effort increases with an increase in complexity $(\tau$ and $\alpha$ ). Similar inferences can be drawn from figure 4 . Thus, the controller gain $K$ is fully dependent on 


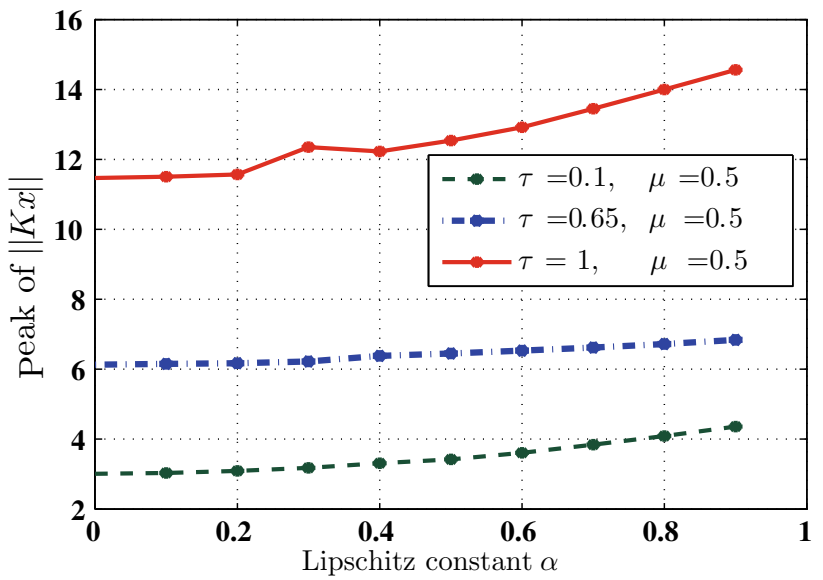

Figure 3. Effect of $\alpha$ on peak of $\|K x\|$.

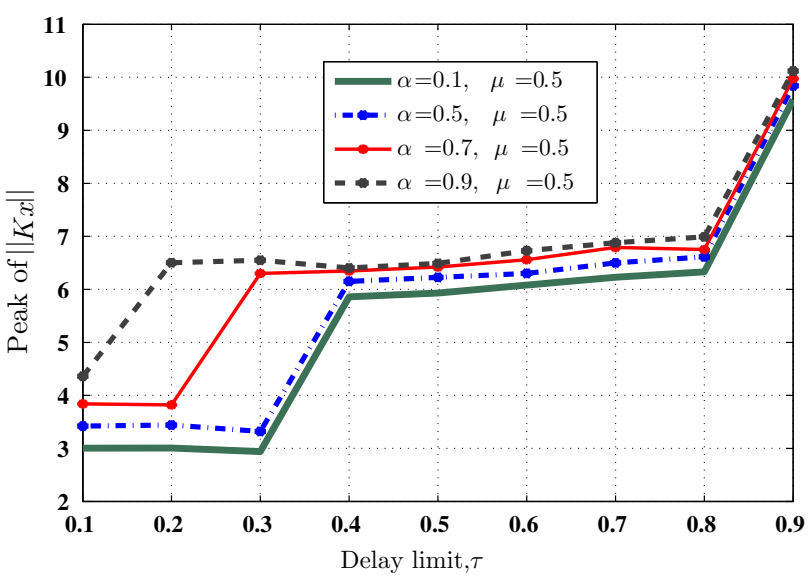

Figure 4. Effect of $\tau$ on peak of $\|K x\|$.

the parameters, i.e., $\tau, \alpha$ and $\mu$, and changes according to the change in these, making the design less conservative.

\section{Conclusion}

An improved result is proposed for delay-dependent stabilization of linear system with time-varying delays. The rate of variation of the time delay is also included as an additional constraint in the proposed theorem, which provides additional information about the system during controller design, thus improving the results. The controller design technique is solely based on a few LMIs, thus making it numerically efficient, easy to design and implement. The proposed controller is tested with the examples present in the literature and comparison tables are given. The approach is further extended to systems having Lipschitz nonlinearities. The LSVF control law may be implemented using observers in situations where states are difficult to access.

\section{Acknowledgements}

This work is financially supported by the Indian Institute of Engineering Science and Technology, Shibpur, West Bengal, India. The authors are grateful to the anonymous reviewers for improving the quality of the paper with their valuable suggestions.

\section{References}

[1] Fridman E 2014 Introduction to time-delay systems-analysis and control. Basel: Springer

[2] Gu K, Chen J and Kharitonov V L 2003 Stability of timedelay systems. Basel: Springer

[3] Sipahi R, Niculescu S I, Abdallah C T, Michiels W and Gu K 2011 Stability and stabilization of systems with time delay. IEEE Control Systems 31(1): 38-65

[4] Kim J H, Jeung E T and Park H B 1996 Robust control for parameter uncertain delay systems in state and control input. Automatica 32(9): 1337-1339

[5] Chen C Y and Lee C H 2009 Delay-independent stabilization of linear systems with time-varying delayed state and uncertainties. Journal of the Franklin Institute 346(4): 378-390

[6] Chen B, Liu X and Tong S 2007 New delay-dependent stabilization conditions of T-S fuzzy systems with constant delay. Fuzzy Sets and Systems 158(20): 2209-2224

[7] Chen W H and Zheng W X 2007 Delay dependent robust stabilization for uncertain neutral systems with distributed delays. Automatica 43(1): 95-104

[8] Li X and De Souza C E 1997 Delay-dependent robust stability and stabilization of uncertain linear delay systems: a linear matrix inequality approach IEEE Transactions on Automatic Control 42(8): 1144-1148

[9] Li X and De Souza C E 1997 Criteria for robust stability and stabilization of uncertain linear systems with state delay. Automatica 33(9): 1657-1662

[10] Moon Y S, Park P, Kwon W H and Lee Y S 2001 Delaydependent robust stabilization of uncertain state-delayed systems. International Journal of Control 74(14): 1447-1455

[11] Fridman E and Shaked U 2002 An improved stabilization method for linear time-delay systems. IEEE Transactions on Automatic Control 47(11): 1931-1937

[12] Zhang X M, Wu M, She J H and He Y 2005 Delay-dependent stabilization of linear systems with time-varying state and input delays. Automatica 41(8): 1405-1412

[13] Wu M, He Y and She J H 2010 Stability analysis and robust control of time-delay systems. Berlin, Heidelberg: Springer

[14] He Y, Wang Q G, Lin C and Wu M 2007 Delay-rangedependent stability for systems with time-varying delay. Automatica 43(2): 371-376

[15] Zeng H B, He Y, Wu M and She J 2015 Free-matrix-based integral inequality for stability analysis of systems with timevarying delay. IEEE Transactions on Automatic Control 60(10): 2768-2772

[16] Xu S, Lam J, Zhang B and Zou Y 2015 New insight into delay-dependent stability of time-delay systems. International Journal of Robust and Nonlinear Control 25(7): 961-970 
[17] Gouaisbaut F and Peaucelle D 2006 Delay-dependent stability analysis of linear time delay systems. IFAC Proceedings Volumes 39(10): 54-59

[18] Kim J H 2001 Delay and its time-derivative dependent robust stability of time-delayed linear systems with uncertainty. IEEE Transactions on Automatic Control 46(5): 789-792

[19] Wu M, He Y, She J H and Liu G P 2004 Delay-dependent criteria for robust stability of time-varying delay systems. Automatica 40(8): 1435-1439

[20] He Y, Wu M, She J H and Liu G P 2004 Parameter-dependent Lyapunov functional for stability of time-delay systems with polytopic-type uncertainties. IEEE Transactions on Automatic Control 49(5): 828-832

[21] Gu K 2000 An integral inequality in the stability problem of time-delay systems In: Proceedings of the 39th IEEE Conference on Decision and Control, vol. 3, pp. 2805-2810

[22] Niculescu S I, Verriest E I, Dugard L and Dion J M 1998 Stability and robust stability of time-delay systems: a guided tour. In: Stability and control of time-delay systems. Berlin: Springer, pp. 1-71

[23] Xu S and Lam J 2007 On equivalence and efficiency of certain stability criteria for time-delay systems. IEEE Transactions on Automatic Control 52(1): 95-101

[24] Lin C, Wang Q G and Lee T H 2006 Delay-dependent LMI conditions for stability and stabilization of T-S fuzzy systems with bounded time-delay. Fuzzy Sets and Systems 157(9): 1229-1247

[25] Cao Y Y and Frank P M 2000 Analysis and synthesis of nonlinear time-delay systems via fuzzy control approach. IEEE Transactions on Fuzzy Systems 8(2): 200-211

[26] Wang M, Chen B, Liu X and Shi P 2008 Adaptive fuzzy tracking control for a class of perturbed strict-feedback nonlinear time-delay systems. Fuzzy Sets and Systems 159(8): 949-967

[27] Ge S S, Hong F and Lee T H 2004 Adaptive neural control of nonlinear time-delay systems with unknown virtual control coefficients. IEEE Transactions on Systems Man and Cybernetics, Part B: Cybernetics 34(1): 499-516

[28] Wang M, Liu X and Shi P 2011 Adaptive neural control of pure-feedback nonlinear time-delay systems via dynamic surface technique. IEEE Transactions on Systems, Man, and Cybernetics, Part B: Cybernetics 41(6): $1681-1692$

[29] Nguang S K 2000 Robust stabilization of a class of timedelay nonlinear systems. IEEE Transactions on Automatic Control 45(4): 756-762

[30] Zhang X, Liu Q, Baron L and Boukas E K 2011 Feedback stabilization for high order feedforward nonlinear time-delay systems. Automatica 47(5): 962-967

[31] Zhang X, Baron L, Liu Q and Boukas E K 2011 Design of stabilizing controllers with a dynamic gain for feedforward nonlinear time-delay systems. IEEE Transactions on Automatic Control 56(3): 692-697

[32] Chen M S and Chen C C 2007 Robust nonlinear observer for Lipschitz nonlinear systems subject to disturbances. IEEE Transactions on Automatic Control 52(12): 23652369

[33] Hassan L, Zemouche A and Boutayeb M 2014 A new observer-based controller design method for a class of timevarying delay systems with Lipschitz nonlinearities. In: Proceedings of the American Control Conference, ACC, pp. 4163-4168

[34] Phanomchoeng G and Rajamani R 2010 Observer design for Lipschitz nonlinear systems using Riccati equations. In: American Control Conference, ACC, pp. 6060-6065

[35] Ali Z and Mohamed B 2013 On LMI conditions to design observers for Lipschitz nonlinear systems. Automatica 49(2): 585-591

[36] Chen Y, Xue A, Lu R and Zhou S 2008 On robustly exponential stability of uncertain neutral systems with timevarying delays and nonlinear perturbations. Nonlinear Analysis: Theory, Methods and Applications 68(8): 2464-2470

[37] Zhang W, Cai X S and Han Z Z 2010 Robust stability criteria for systems with interval time-varying delay and nonlinear perturbations. Journal of Computational and Applied Mathematics 234(1): 174-180

[38] Liu L P 2009 Robust exponential stability for uncertain timevarying delay systems with delay dependence. Journal of the Franklin Institute 346(10): 958-968 